PROCEEDINGS OF THE

AMERICAN MATHEMATICAL SOCIETY

Volume 131, Number 3, Pages 701-708

S 0002-9939(02)06575-9

Article electronically published on September 17, 2002

\title{
WHAT IS THE REES ALGEBRA OF A MODULE?
}

\author{
DAVID EISENBUD, CRAIG HUNEKE, AND BERND ULRICH
}

(Communicated by Wolmer V. Vasconcelos)

\begin{abstract}
In this paper we show that the Rees algebra can be made into a functor on modules over a ring in a way that extends its classical definition for ideals. The Rees algebra of a module $M$ may be computed in terms of a "maximal" map $f$ from $M$ to a free module as the image of the map induced by $f$ on symmetric algebras. We show that the analytic spread and reductions of $M$ can be determined from any embedding of $M$ into a free module, and in characteristic 0 - but not in positive characteristic! - the Rees algebra itself can be computed from any such embedding.
\end{abstract}

The Rees algebra of an ideal $I$ in a ring $R$, namely $\mathcal{R}=\bigoplus_{n=0}^{\infty} I^{n}=R[I t] \subset$ $R[t]$, plays a major role in commutative algebra and in algebraic geometry since $\operatorname{Proj}(\mathcal{R})$ is the blowup of $\operatorname{Spec}(R)$ along the subscheme defined by $I$. Several authors have found it useful to generalize this construction from ideals to modules; see for instance Gaffney and Kleiman GK, Katz [ ], Katz and Kodiyalam [KK, Kleiman and Thorup [KT], Kodiyalam [Ko, Liu [L], Rees [R], Simis, Ulrich, and Vasconcelos [SUV1], SUV2], and Vasconcelos [V], who define the Rees algebra of a module satisfying one or another hypothesis. Usually this hypothesis was tailored to approach the problem(s) the authors were interested in solving.

The goal of this paper is to clarify the definition for arbitrary finitely generated modules over a Noetherian ring $R$. Our interest in this clarification arose through our work on generalized prinicipal ideal theorems and the heights of ideals of minors, where we heavily use Rees algebras (see Eisenbud-Huneke-Ulrich [EHU1, EHU2]). It seems worthwhile to understand the differences and similarities of the various approaches from the papers above, and to make the definition as functorial as possible. Even for ideals there is a problem: in the grade 0 case it is not clear from the definition above whether the Rees algebra depends on the embedding of $I$ in $R$.

A natural approach is to define the Rees algebra of a module as the symmetric algebra modulo $R$-torsion (that is, modulo elements killed by non-zerodivisors of $R$ ). This does not provide a satisfactory definition in all cases in the sense that it may give the wrong answer even for an ideal, if the ring is not a domain. In general, as was well-known, it is a good definition when the module $M$ "has a rank", i.e., when $M$ is free of constant rank locally at the associated primes of $R$. This hypothesis is sufficient for many applications; however, for example, it is

Received by the editors May 2, 2001 and, in revised form, October 19, 2001.

2000 Mathematics Subject Classification. Primary 13A30, 13B21; Secondary 13C12.

Key words and phrases. Rees algebra, module, integral dependence.

All three authors were partially supported by the NSF.

(C)2002 American Mathematical Society 
not necessarily preserved replacing $M$ by $M / x M$ and $R$ by $R / x R$ even if $x$ is a non-zerodivisor on $M$ and $R$.

Another alternative is to consider a module $M$ together with an embedding into a free module $G$, and define the Rees algebra of $M$ to be the subalgebra of the symmetric algebra of $G$ generated by $M$. More generally, for any homomorphism $g: M \rightarrow N$ we define $\mathcal{R}(g)$, the Rees algebra of $g$, to be the graded $R$-algebra which is the image of the map $\operatorname{Sym}(g): \operatorname{Sym}(M) \rightarrow \operatorname{Sym}(N)$. One may then try to define the Rees algebra of $M$ as $\mathcal{R}(g)$ for an embedding $g$ of $M$ into a free module $G$.

However, the result may depend on the chosen embedding $g$. In Section 1 we give as example a principal ideal $I$ in an Artinian ring of characteristic $p>0$, and an embedding $g: I \rightarrow R^{2}$ such that $\mathcal{R}(g)$ is not isomorphic to $\mathcal{R}(I)=\bigoplus_{n=0}^{\infty} I^{n}$. Of course $\mathcal{R}(I)$ may also be expressed as $\mathcal{R}(i)$, where $i$ is the inclusion of $I$ as an ideal of $R$. Thus $\mathcal{R}(g)$ depends on $g$, not only on $M$.

Here we take a third alternative:

Definition 0.1. If $R$ is a ring and $M$ is an $R$-module, we define the Rees algebra of $M$ to be

$$
\mathcal{R}(M)=\operatorname{Sym}(M) /\left(\bigcap_{g} L_{g}\right)
$$

where the intersection is taken over all homomorphisms $g$ from $M$ to free $R$-modules, and $L_{g}$ denotes the kernel of $\operatorname{Sym}(g)$.

Although the definition may at first appear somewhat complicated, it is at least obviously functorial: if $h: M \rightarrow N$ is a homomorphism of $R$-modules, then for every homomorphism from $N$ to a free module $g: N \rightarrow G$ the map $g h$ is a homomorphism from $M$ to a free module, $\operatorname{so} \operatorname{Sym}(h): \operatorname{Sym}(M) \rightarrow \operatorname{Sym}(N)$ induces an $R$-algebra homomorphism $\mathcal{R}(M) \rightarrow \mathcal{R}(N)$. As the symmetric algebra functor preserves epimorphisms, so does the Rees algebra functor.

In Section 1 we solve the problem of computing $\mathcal{R}(M)$ (if $M$ is finitely generated) by showing that $\mathcal{R}(M)=\mathcal{R}(f)$ for any homomorphism from $M$ to a free module $F$ such that the dual map $F^{*} \rightarrow M^{*}$ is surjective. This implies that forming the Rees algebra of a finitely generated module over a Noetherian ring commutes with flat base change. We also show that if $g: M \rightarrow R$ is an embedding in a free module of rank 1 , then $\mathcal{R}(M)=\mathcal{R}(g)$, so that $\mathcal{R}(M)$ agrees with the classical definition for ideals (in particular, this shows that the classical definition is independent of the choice of representation of $M$ as an ideal). Moreover, we show that in many cases $\mathcal{R}(M)$ can be computed from any embedding. The following is a special case of what we prove:

Theorem 0.2. Let $R$ be a Noetherian ring and let $M$ be a finitely generated $R$ module. If $R$ is torsion free over $\mathbb{Z}$, or $R$ is unmixed and generically Gorenstein, or $M$ is free locally at each associated prime of $R$, then $\mathcal{R}(M) \cong \mathcal{R}(g)$ for any embedding $g: M \rightarrow G$ of $M$ into a free module $G$.

In Section 2 we use our Rees algebra construction to introduce analytic spread and integral dependence for arbitrary modules. We prove that for any embedding $g$ of $M$ into a free module, the natural map $\mathcal{R}(M) \rightarrow \mathcal{R}(g)$ has nilpotent kernel. It follows that analytic spread and integral dependence can be determined in $\mathcal{R}(g)$.

In two subsequent papers [EHU1], [EHU2] we apply the notions developed here to obtain new generalized principal ideal theorems and results on heights of ideals of minors of a matrix. 


\section{REes ALGEBRAS}

We begin with an example showing that the Rees algebra of a module cannot be defined from an arbitrary embedding into a free module, even when the module is an ideal:

Example 1.1. Let $k$ be a field of characteristic $p$, let

$$
R=k[X, Y, Z] /\left(\left(X^{p}, Y^{p}\right)+(X, Y, Z)^{p+1}\right),
$$

write $x, y, z$ for the images of $X, Y, Z$ in $R$, and take $M$ to be the ideal $M=$ $R z \cong R /(x, y, z)^{p}$. Write $g_{1}: M \rightarrow R$ for the inclusion $M=R z \subset R$, and let $g_{2}: M \rightarrow R^{2}=R t_{1} \oplus R t_{2}$ be the homomorphism sending $z$ to $x t_{1}+y t_{2}$. It is easy to see that $g_{2}$ is also an embedding. The algebra $\mathcal{R}\left(g_{1}\right)$ is the same as the classical Rees algebra $\mathcal{R}=\bigoplus_{n=0}^{\infty}\left(z^{n}\right)$, and has $p^{\text {th }}$ graded component $\left(z^{p}\right) \neq 0$. On the other hand

$$
\mathcal{R}\left(g_{2}\right)_{p}=R\left(x^{p} t_{1}^{p}+y^{p} t_{2}^{p}\right)=0,
$$

and it follows that $\mathcal{R}\left(g_{2}\right)$ cannot surject onto the classical $\mathcal{R}=\mathcal{R}\left(g_{1}\right)$ by any graded homomorphism, so $\mathcal{R}\left(g_{2}\right) \neq \mathcal{R}(M)$ as graded rings.

To compute the Rees algebra of a module, we use the following notion.

Definition 1.2. Let $R$ be a ring and let $M$ be an $R$-module. We say that $f: M \rightarrow$ $F$ is a versal map to a free module, if $F$ is a free $R$-module, $f$ is a homomorphism, and every homomorphism from $M$ to a free module factors through $f$.

It follows at once from the definition that if $f: M \rightarrow F$ is a versal map to a free $R$-module $F$, then $\mathcal{R}(M)=\mathcal{R}(f)$. With a finiteness assumption it is easy to find such a map:

Proposition 1.3. Let $R$ be a ring and let $M$ be a finitely generated $R$-module. Let $f: M \rightarrow F$ be a homomorphism to a free $R$-module $F$ so that the dual map $F^{*} \rightarrow M^{*}$ is surjective (such $f$ can be obtained by composing the natural map $M \rightarrow M^{* *}$ with the dual of any epimorphism from a finitely generated free module onto $\left.M^{*}\right)$. One has that $f$ is versal and $\mathcal{R}(M)=\mathcal{R}(f)$. In particular, formation of the Rees algebra of a finitely generated module over a Noetherian ring commutes with flat base change.

Proof. Let $g: M \rightarrow G$ be a homomorphism to a free $R$-module. We must show that $g$ factors through $f$. Since $M$ is finitely generated, $g$ factors through a finitely generated free summand of $G$, and we may assume that $G$ is finitely generated. It follows that the dual $G^{*}$ is free. Consequently we may write $g^{*}=f^{*} h$. We may also suppose that $F$ is finitely generated. Since $F$ and $G$ are reflexive, the desired factorization is $g=h^{*} f$.

The equality $\mathcal{R}(M)=\mathcal{R}(f)$ has been observed just before the Proposition. Finally assume that $R$ is Noetherian and let $f$ be any homomorphism satisfying our hypothesis. If $S$ is a flat $R$-algebra, then $\operatorname{Hom}_{S}\left(S \otimes_{R} M, S\right)=S \otimes_{R} \operatorname{Hom}_{R}(M, R)$ because $M$ is finitely presented. Thus $S \otimes_{R} f$ has a surjective $S$-dual, and it follows that $\mathcal{R}\left(S \otimes_{R} M\right)=\mathcal{R}\left(S \otimes_{R} f\right)=S \otimes_{R} \mathcal{R}(f)=S \otimes_{R} \mathcal{R}(M)$, as required.

Example 1.1 continued. By Proposition 1.3 the map $g_{2}$ is not versal. It is easy to check that $M^{*}$ requires 3 generators and a versal map from $M$ to $R^{3}$ may be written as

$$
f: M=R z \rightarrow R^{3}=R t_{1} \oplus R t_{2} \oplus R t_{3}, \quad z \mapsto x t_{1}+y t_{2}+z t_{3} .
$$


We have

$$
\mathcal{R}(f)_{p}=R\left(x^{p} t_{1}^{p}+y^{p} t_{2}^{p}+z^{p} t_{3}^{p}\right)=R z^{p} t_{3}^{p}=\mathcal{R}(M)_{p},
$$

as implied by Proposition 1.3.

Next we show that our definition of the Rees algebra agrees with the classical notion for ideals, which is thus independent of the embedding of the ideal into $R$.

Theorem 1.4. Let $R$ be a ring, let $M$ be a finitely generated ideal of $R$, and let $g: M \rightarrow R$ be the inclusion map. The natural map from $\mathcal{R}(M)$ to the classical Rees algebra $\mathcal{R}=\bigoplus_{n=0}^{\infty} I^{n}=\mathcal{R}(g)$ is an isomorphism.

Proof. Let $f: M \rightarrow F$ be a versal map to a free module $F$, of rank $n$, say. Since $f$ is versal we may find a homomorphism $h: F \rightarrow R$ so that $g=h f$. We must show that $\phi:=\operatorname{Sym}(h)$ is a monomorphism on the subring of $\operatorname{Sym}(F)$ generated by $f(M)$.

Write $\operatorname{Sym}(F)=R\left[t_{1}, \ldots, t_{n}\right]$, and $\operatorname{Sym}(R)=R[z]$. Let $m_{1}, \ldots, m_{s}$ be generators of $M$, and write $a_{i}=g\left(m_{i}\right) \in R$, so that $\phi\left(f\left(m_{i}\right)\right)=a_{i} z$. Let $S=R\left[x_{1}, \ldots, x_{s}\right]$ be a polynomial ring, and consider the homomorphism $\psi: S \rightarrow \operatorname{Sym}(F)$ sending $x_{i}$ to $f\left(m_{i}\right)$. We must show that the kernel of $\psi$ is the same as the kernel of $\phi \psi$. Giving each $x_{i}$ degree 1, the kernel of $\phi \psi$ is homogeneous, so it suffices to show that if $u \in S$ is a form of degree $d$ such that $\phi \psi(u)=0$, then $\psi(u)=0$. We do induction on $d$, the case $d=0$ being obvious.

We may write $u=\sum_{i=1}^{s} x_{i} u_{i}$ where the $u_{i}$ are forms of degree $d-1$. We see that

$$
0=\phi \psi(u)=\phi \psi\left(\sum_{i} x_{i} u_{i}\right)=\phi \psi\left(\sum_{i} a_{i} u_{i}\right) z,
$$

so $\phi \psi\left(\sum_{i} a_{i} u_{i}\right)=0$. By our induction hypothesis, $\psi\left(\sum_{i} a_{i} u_{i}\right)=0$, too.

We may expand each $\psi\left(u_{i}\right)$ in the form $\psi\left(u_{i}\right)=\sum_{\alpha} r_{i, \alpha} t^{\alpha}$, where the sum runs over all multi-indices $\alpha$ of weight $d-1$, and thus $\sum_{i} \sum_{\alpha} a_{i} r_{i, \alpha} t^{\alpha}=0$. Since the distinct monomials $t^{\alpha}$ are linearly independent, we have $\sum_{i} a_{i} r_{i, \alpha}=0$ for each $\alpha$. By our hypothesis that $g$ is an embedding, the $f\left(m_{i}\right)$ satisfy the same linear relations as the $a_{i}$, so we get $\sum_{i} f\left(m_{i}\right) r_{i, \alpha}=0$ for each $\alpha$, and finally $\psi(u)=$ $\sum_{i} \sum_{\alpha} f\left(m_{i}\right) r_{i, \alpha} t^{\alpha}=0$, as required.

For the proofs that follow we need to identify the minimal and the associated primes of $\mathcal{R}(M)$.

Proposition 1.5. Let $R$ be a Noetherian ring and let $M$ be a finitely generated $R$-module. There is a one-to-one correspondence between the associated primes of $\mathcal{R}(M)$ and the associated primes of $R$ given by $P \mapsto R \cap P$, and likewise for minimal primes.

Proof. Notice that $\mathcal{R}(M)$ is an $R$-subalgebra of a polynomial ring $S$ in finitely many variables over $R$. Every associated prime of $R$ is the contraction of an associated prime of $\mathcal{R}(M)$, and every associated prime of $\mathcal{R}(M)$ is the contraction of an associated prime of the polynomial ring $S$, which in turn is extended from an associated prime of $R$. Since the resulting one-to-one correspondence between $\operatorname{Ass}(\mathcal{R}(M))$ and $\operatorname{Ass}(R)$ is order preserving, we have also proved the claim about minimal primes.

Note that a versal map from a finitely generated module $M$ to a free module has the same image as the natural map from $M$ to its double dual. This image is 
called the torsionless quotient of $M$. Any homomorphism from $M$ to a free module factors uniquely through the torsionless quotient of $M$. The following result gives conditions under which the Rees algebra of a torsionless module can be deduced from any inclusion into a free module. For convenience in applications we will state it without the torsionless hypothesis.

Theorem 1.6. Let $R$ be a Noetherian ring, let $M$ be a finitely generated $R$-module, and let $g: M \rightarrow G$ be a homomorphism to a free $R$-module $G$ inducing an inclusion on the torsionless quotient of $M$. If for each associated prime $Q$ of $R$ either $R_{Q}$ is Gorenstein, or $M_{Q}$ is free, or $R_{Q}$ is $\mathbb{Z}$-torsion free, then the natural epimorphism $\mathcal{R}(M) \rightarrow \mathcal{R}(g)$ is an isomorphism.

Proof. Replacing $M$ by its torsionless quotient, we may assume that $g: M \rightarrow G$ is an inclusion.

Let $f: M \rightarrow F$ be a versal map from $M$ to a free module, and let $\mathcal{R}=\mathcal{R}(M)=$ $\mathcal{R}(f)$ be the Rees algebra of $M$. Let $h: F \rightarrow G$ be a homomorphism with $g=h f$ and let $\phi: \mathcal{R} \rightarrow \mathcal{R}(g)$ be the induced epimorphism. By Proposition 1.5 every associated prime of $\mathcal{R}$ contracts to an associated prime of $R$. Hence to prove the injectivity of $\phi$ we may replace $R$ by $R_{Q}$, where $Q$ is an associated prime of $R$.

If $R$ is Gorenstein (and hence Artinian), then free modules are injective, and therefore any monomorphism from a module to a free module is versal. Similarly, if $M$ is free, then since $R$ has depth 0 , any monomorphism from $M$ to a free module splits, and thus again is versal. In either case we see that $f$ and $g$ are both versal, and the injectivity of $\phi$ follows from the functoriality of the Rees algebra.

Finally, we treat the case where $R$ is $\mathbb{Z}$-torsion free. It suffices to consider the case where $F=G \oplus H$ is finitely generated with $H$ free, and $h$ is the natural projection. Set $J=H \cdot \operatorname{Sym}(F)$, the kernel of $\operatorname{Sym}(h)$. Since $g$ is an inclusion, $\phi$ is an injection in degree 1 , and we must show that $\phi$ is an injection in every degree, or equivalently $J \cap \mathcal{R}=0$. This follows from the next Lemma:

Lemma 1.7. Let $R$ be a ring and let $F$ be a finitely generated free $R$-module. Let $M$ be a submodule of $F$, and let $\mathcal{R}$ be the subalgebra of $\operatorname{Sym}(F)$ generated by $M$. Let $H$ be a summand of $F$ with $H \cap M=0$. If $d$ ! is a non-zerodivisor in $R$, then $(H \cdot \operatorname{Sym}(F)) \cap \mathcal{R}_{d}=0$.

Proof. It is enough to prove that the Lemma holds after localizing at each maximal ideal of $R$, so we may assume that $R$ is local.

Let $t_{1}, \ldots, t_{n}$ be a basis of $F$. Since $R$ is local we may suppose that $H$ is generated by $t_{m+1}, \ldots, t_{n}$. Set $J=H \cdot \operatorname{Sym}(F)$. We may assume that $d>1$. Since $(d-1)$ ! is a non-zerodivisor as well, we know by induction that $J \cap \mathcal{R}_{d-1}=0$. Writing $\partial_{i}=\frac{\partial}{\partial t_{i}}$, one has $\partial_{i}(\mathcal{R}) \subset \mathcal{R}$ for every $i$ because the $R$-algebra $\mathcal{R}$ is generated by linear forms, $\partial_{i}(J) \subset J$ for every $i \leq m$, and $\partial_{i}\left(J^{2}\right) \subset J$ for every $i$.

Now if $u \in J \cap \mathcal{R}_{d}$, then $\partial_{i}(u) \in J \cap \mathcal{R}_{d-1}=0$ for every $i \leq m$. Since $d$ ! is a non-zerodivisor on $R$, it follows that $u \in R\left[t_{m+1}, \ldots, t_{n}\right]_{d} \subset J^{2}$. Thus $\partial_{i}(u) \in$ $J \cap \mathcal{R}_{d-1}=0$ for every $i$, and hence $u=0$ because $d$ ! is a non-zerodivisor.

To connect our definition of the Rees algebra with the torsion in the symmetric algebra, suppose that $R$ is a Noetherian ring, $M$ is a finitely generated $R$-module, and $\mathcal{A}$ is the $R$-torsion of $\operatorname{Sym}(M)$. If $M_{Q}$ is free for every associated prime $Q$ of $R$ and if $f: M \rightarrow F$ is a versal map to a free module, then $\operatorname{Sym}(f)$ induces an 
isomorphism $\operatorname{Sym}(M) / \mathcal{A} \sim \mathcal{R}(M)$. This follows from Proposition 1.5 and the fact that $\operatorname{Sym}(f)_{Q}$ is injective for every associated prime $Q$ of $R$.

If we are only interested in the reduced structure of the Rees algebra of $M$, then we can compute it from any embedding of the torsionless quotient of $M$.

Proposition 1.8. Let $R$ be a Noetherian ring, let $M$ be a finitely generated $R$ module, and let $g: M \rightarrow G$ be a homomorphism to a free $R$-module $G$ inducing an inclusion on the torsionless quotient of $M$. The kernel of the natural epimorphism $\mathcal{R}(M) \rightarrow \mathcal{R}(g)$ is nilpotent.

Proof. By Proposition 1.5 every minimal prime of $\mathcal{R}(M)$ contracts to a minimal prime of $R$, so it is enough to prove the result after localizing at a minimal prime of $R$. Thus we may assume that $(R, \mathbf{m})$ is Artinian and local. We may also suppose that $G$ is finitely generated and we may replace $M$ by its torsionless quotient to assume that $g$ is a monomorphism.

Let $f: M \rightarrow F$ be a versal map from $M$ to a free module, and let $K$ be the kernel of the natural epimorphism $\mathcal{R}(M)=\mathcal{R}(f) \rightarrow \mathcal{R}(g)$. Suppose first that $M$ has no free summand. Because $R$ is local, it follows that $\operatorname{Im} f \subset \mathbf{m} F$. The kernel $K$ must be contained in the positive degree part of $\mathcal{R}(M)$, which is contained in $\mathbf{m} \operatorname{Sym}(F)$. As $\mathbf{m}$ is nilpotent, $K$ is nilpotent as well.

In the general case, let $H$ be a maximal free submodule of $M$. Since any inclusion of finitely generated free modules over an Artinian ring splits, we may write $G=$ $G^{\prime} \oplus H$ and $M=M^{\prime} \oplus H$ in such a way that $g=g^{\prime} \oplus 1_{H}$. The map $\mathcal{R}(M) \rightarrow \operatorname{Sym}(G)$ is obtained from the map $\mathcal{R}\left(M^{\prime}\right) \rightarrow \operatorname{Sym}\left(G^{\prime}\right)$ induced by $g^{\prime}$ by adjoining polynomial variables. As the kernel of the latter map is nilpotent, the desired result follows.

\section{INTEGRAL DEPENDENCE}

In this section we introduce general definitions of integral dependence and of analytic spread for modules that we will apply elsewhere.

Definition 2.1. Let $R$ be a ring, $M$ an $R$-module, and $U \subset L$ submodules of $M$.

(1) Let $U^{\prime}, L^{\prime}$ be the images of $U, L$ in $\mathcal{R}(M)$ and consider the subalgebras $R\left[U^{\prime}\right] \subset R\left[L^{\prime}\right] \subset \mathcal{R}(M)$. We say $L$ is integral over $U$ in $M$ if the ring extension $R\left[U^{\prime}\right] \subset R\left[L^{\prime}\right]$ is integral.

(2) We say $M$ is integral over $U$ or $U$ is a reduction of $M$, if $M$ is integral over $U$ in $M$.

In the situation of Definition 2.1(1) the Rees algebra of $L$ maps to the Rees algebra of $M$, so if $L$ is integral over $U$, then $L$ is integral over $U$ in $M$.

Theorem 2.2. Let $R$ be a Noetherian ring, $M$ a finitely generated $R$-module, $U \subset L$ submodules of $M$, and $f: M \rightarrow F$ a versal map from $M$ to a free $R$-module. The following are equivalent:

(1) $L$ is integral over $U$ in $M$.

(2) For every minimal prime $Q$ of $R$, the module $L^{\prime}$ is integral over $U^{\prime}$ in $M^{\prime}$, where' denotes images in $F / Q F$.

(3) For every homomorphism $M \rightarrow G$ to a free $R$-module and for every homomorphism $R \rightarrow S$ to a domain $S$, the module $L^{\prime}$ is integral over $U^{\prime}$ in $M^{\prime}$, where' denotes tensoring with $S$ and taking images in $S \otimes_{R} G$. 
(4) For every homomorphism $R \rightarrow V$ to a rank one discrete valuation ring $V$ whose kernel is a minimal prime of $R$, we have $U^{\prime}=L^{\prime}$, where' denotes tensoring with $V$ and taking images in $V \otimes_{R} F$.

(5) (Valuative Criterion of Integrality) For every homomorphism $M \rightarrow G$ to a free $R$-module and every homomorphism $R \rightarrow V$ to a rank one discrete valuation ring $V$, we have $U^{\prime}=L^{\prime}$, where' denotes tensoring with $V$ and taking images in $V \otimes_{R} G$.

Proof. By the functoriality of the Rees algebra, we may assume $G=F$ in parts (3) and (5). As $\mathcal{R}(M)$ embeds into $\mathcal{R}(F)=\operatorname{Sym}(F)$, we may replace $M$ by $F$ in (1). In parts (2)-(5), the rings $R / Q, S, V$ are domains, and hence by Theorem 1.6 the embedding of $L^{\prime}$ and $M^{\prime}$ into the free modules $F / Q F, S \otimes_{R} F, V \otimes_{R} F$, respectively, can be used to define $\mathcal{R}\left(L^{\prime}\right)$ and $\mathcal{R}\left(M^{\prime}\right)$. In particular we may replace $M$ by $F$ in these parts as well.

Now it is obvious that (1) implies (3). Part (1) follows from (2) since $\mathcal{R}(F) / \sqrt{0} \subset$ $\prod_{Q} \mathcal{R}(F / Q F)$, where $Q$ ranges over all minimal primes of $R$ and $F / Q F$ is considered as a module over $R / Q$. Finally, the equivalence of (2) and (4) and of (3) and (5) has been shown in Rees [R, 1.5(ii)].

We see from Theorem 2.2 that our definition of integrality differs from that of Rees $[\mathrm{R}$, p. 435] when $R$ is not a domain. Rees' definition amounts to saying that for every minimal prime $Q$ of $R$, the module $L^{\prime}$ is integral (in our sense or his) over $U^{\prime}$ in $M^{\prime}$, where now ' denotes images in $M / Q M$. If, for example, $k$ is a field, $R=k[x] /\left(x^{2}\right)$, and $U=0 \subset L=M=(x) /\left(x^{2}\right)$, then $M$ is integral over $U$ in our sense but not in the sense of Rees.

Definition 2.3. Let $R$ be a local ring with residue field $k$ and let $M$ be a finitely generated $R$-module. The analytic spread $\ell(M)$ of $M$ is the Krull dimension of $k \otimes_{R} \mathcal{R}(M)$.

By way of illustration, we remark that the analytic spread of a finitely generated module $M$ over an Artinian local $\operatorname{ring}(R, \mathbf{m}, k)$ is equal to the rank $r$ of a maximal free summand $H$ of $M$ (and is also equal to the dimension of the Rees algebra of the module). Indeed, writing $M=M^{\prime} \oplus H$ we have that any homomorphism from $M$ to a free module $F$ carries $M^{\prime}$ into $\mathbf{m} F$, which generates a nilpotent ideal of $\operatorname{Sym}(F)$. Thus $(\mathcal{R}(M) / \mathbf{m} \mathcal{R}(M))_{\text {red }}=\mathcal{R}(M)_{\text {red }}$ is a polynomial ring over $k$ in $r$ variables.

If $k$ is infinite one can show as in the case of ideals, using a homogeneous Noether normalization of $k \otimes_{R} \mathcal{R}(M)$ and Nakayama's Lemma, that

$$
\ell(M)=\min \{\mu(U) \mid U \text { is a reduction of } M\} .
$$

Furthermore, $\ell(M) \leq \mu(M)$ and equality holds if and only if $M$ has no proper reduction.

Proposition 2.4. Let $R$ be a Noetherian local ring with residue field $k$, let $M$ be a finitely generated $R$-module, and let $g: M \rightarrow G$ be a homomorphism to a free $R$-module $G$. If $g$ induces an inclusion on the torsionless quotient of $M$, then $\ell(M)=\operatorname{dim} k \otimes_{R} \mathcal{R}(g)$.

Proof. Proposition 1.8 shows that $\mathcal{R}(M)$ differs from $\mathcal{R}(g)$ only by a nilpotent ideal, and thus the same holds after tensoring with $k$. 


\section{BIBLIOGRAPHY}

[EHU1] D. Eisenbud, C. Huneke and B. Ulrich, Order ideals and a generalized Krull height theorem, to appear in Math. Ann.

[EHU2] — Heights of ideals of minors, preprint, 2001.

[GK] T. Gaffney and S. Kleiman, Specialization of integral dependence for modules, Invent. Math. 137 (1999), 541-574. MR 2000k:32025

[K] D. Katz, Reduction criteria for modules, Comm. in Algebra 23 (1995), 4543-4548. MR 96j: 13022

[KK] D. Katz and V. Kodiyalam, Symmetric powers of complete modules over a twodimensional regular local ring, Trans. Amer. Math. Soc. 349 (1997), 747-762. MR 97g:13041

[KT] S. Kleiman and A. Thorup, Conormal geometry of maximal minors, J. Algebra 230 (2000), 204-221. MR 2001h:13006

[Ko] V. Kodiyalam, Integrally closed modules over two-dimensional regular local rings, Trans. Amer. Math. Soc. 347 (1995), 3551-3573. MR 95m:13015

[L] J.-C. Liu, Rees algebras of finitely generated torsion-free modules over a two-dimensional regular local ring, Comm. in Algebra 26 (1998), 4015-4039. MR 99k:13003

[R] D. Rees, Reduction of modules, Math. Proc. Camb. Phil. Soc. 101 (1987), 431-449. MR 88a:13001

[SUV1] A. Simis, B. Ulrich and W. V. Vasconcelos, Codimension, multiplicity and integral extensions, Math. Proc. Camb. Phil. Soc. 130 (2001), 237-257. MR 2002c:13017

[SUV2] _ Rees algebras of modules, to appear in Proc. London Math. Soc.

[V] W. V. Vasconcelos, Arithmetic of Blowup Algebras, London Math. Soc. Lect. Notes, vol. 195, Cambridge University Press, Cambridge, 1994. MR 95g:13005

Mathematical Sciences Research Institute, 1000 Centennial Dr., Berkeley, CaliforNIA 94720

E-mail address: de@msri.org

Department of Mathematics, University of Kansas, Lawrence, Kansas 66045

E-mail address: huneke@math.ukans.edu

Department of Mathematics, Purdue University, West Lafayette, Indiana 47907

E-mail address: ulrich@math.purdue.edu 